terminus of the 46 residue molecule is placed outside and the basic groups near the C-terminus are arranged inside to neutralise the nucleic acid core. Following criteria worked out by Smillie and his colleagues (Hodges et al., Cold Spring Harb. Symp. quant. Biol., 37, $299 ; 1972$ ) for "knobs-into-holes" interlocking of the tropomyosin side chains in a coiled-coil, the Pf 1 subunit can be arranged so that most of the hydrophobic residues make favourable van der Waals contacts with those on neighbouring $\alpha$ helices. It looks as if this ingenious model building from sequence data refined against a low-resolution Xray fibre pattern may lead to as clear a picture of the atomic structure of this virus as protein crystallographers now produce by automated analysis of high resolution diffraction data from isomorphous crystals.

Structural molecular biology started with model building. Pauling and Corey discovered the $\alpha$ helix in 1951 by folding a stereochemically correct polypeptide chain to optimise the interactions between turns. Crick looked at the interactions between chains and constructed his models for $\alpha$-helical coiled coils as well as his half of the double helix in 1953. Models of these helical molecules could be built and refined since the backbone folding was seen directly in the X-ray fibre diagrams. The chain folding in globular proteins is not, however, apparent from inspection of the forest of spots in single crystal diffraction patterns. Another breakthrough in 1953 was Perutz's introduction of the isomorphous replacement method which led the way for development of protein crystallography from what he called (in a different context) "the ingenious puzzlesolving of the early pioneers to the almost blindfold automation of the present day". The structures of many soluble globular proteins which yield excellent crystals have now been solved to atomic resolution by this technique. Crystallographic scrutiny has not been comparably successful with structural proteins since good crystals with small unit cells are uncommon-perhaps because these proteins are designed to aggregate in their own way. Isometric virus particles, enzyme complexes and other globular protein assemblies have been crystallised and these structures may be solved by crystallographic methods in spite of the very large unit cells. Structural proteins with recognisable backbones-such as tropomyosin filaments which give relatively poor crystals - will be solved like the filamentous phage by refining a molecular model against low resolution $\mathrm{X}$-ray data.

The structure of a virus or a cellular assembly embodies the rules for its formation. The filamentous bacterio-

\section{Visions of a laser accelerator}

\author{
from O. S. Heavens
}

A LiTTLE over twenty years ago, Smith and Purcell observed the emission of light from a metallised diffraction grating when a $300 \mathrm{keV}$ beam of electrons was directed along the surface in a direction perpendicular to the rulings. This phenomenon, which is referred to as the Smith-Purcell effect, is easily understood in terms of the oscillations induced in the charge carriers in the grating consequent upon the passage of electrons close to the surface. Oscillations occur, minute dipoles are created, and for the appropriate choice of electron velocity the radiation emitted will lie in the visible spectrum. The effect has attracted a modest interest and several papers have appeared giving detailed theoretical accounts of the effect, which can be understood conveniently in terms of leaky spacecharge waves from periodic structure, generally considered as alternately conducting and insulating strips.

Curiously enough, the emphasis has been entirely on the radiation produced by the interaction of the electron beam with the structure. The reverse effect-in which we ask what would happen to an electron beam near a periodic structure when an electromagnetic wave passes over the grating-seems not to have been considered until recently (Mizuno, Ono and Shimoe: this issue of Nature, page 184). The intriguing possibility arises of using a coherent (laser) beam in such a way as to produce an accelerated beam of electrons. Accelerations of the order $1 \mathrm{GeV}$ per metre seem to be feasible, coupled with the possibility of current pulses as short as $10^{-15}$ second, thus opening a new time domain to the experimentalist. It may be early to speculate that the typical large, expensive particle accelerator will become a white elephant, displaced by a laser of relatively trifling cost and an old diffraction grating. If this happens, and if laser fusion becomes a reality, optics may cease to be dismissed as an unfashionable old-hat topic neatly finished by one J. C. Maxwell!

phage is not constructed by simple selfassembly of coat protein and nucleic acid even though the design of the particle is determined by the specific bonding properties of its parts. A regulatory virus protein combines with the viral DNA strand in the cytoplasm thereby segregating it from the replicating circular duplex and controlling its synthesis as a separate circle. The coat protein does not appear in a soluble form in the cytoplasm but is incorporated as it is made into the plasma membrane of the bacterium. Particle assembly proceeds by extrusion of the pre-packaged circular DNA molecule through the membrane bilayer where coat protein displaces regulatory protein. The assembly process is reversed in infection. Following attachment to a susceptible host by the A-protein located at one end of the virus filament, the coat protein dissolves in the membrane bilayer as the DNA and Aprotein are transported into the bacterium.

In the bacterial membrane, the virus coat protein may still be folded in its $\alpha$-helical conformation stabilised by the oily environment; and it may span the bilayer with its positive and negative ends on opposite sides. This picture is reminiscent of many speculative models for membrane proteins-and one recent experimental one. Richard Henderson at the MRC laboratory in Cambridge has recently seen by X-ray diffraction that the purple membrane protein of Halobacterium halobium is folded into $\alpha$-helical segments directed approximately normal to the plane of the bilayer and these segments appear to be packed together with coiled-coil interlocking (J. molec. Biol., in the press).

The filamentous bacteriophage in its different stages of development may be as useful for understanding membrane differentiation as it will be for comprehending fibrous protein organisation, regulation of DNA synthesis and control of virus assembly. Marvin has revealed this structure as a microcosm in which these basic problems in structural biology can be critically explored.

\section{Molecular weight distribution in polymers}

\author{
from Paul Calvert
}

GEL permeation chromatography (GPC) is the only satisfactory method of studying molecular weight distribution in synthetic polymers. Recent improvements in column packing materials and in molecular weight sensitive detectors should allow the development of this technique from a slow, poorly calibrated measurement into a rapid. accurate one.

Synthetic polymers owe their utility to their high molecular weight, and average molecular weight is a much measured characteristic. But some important 